\title{
Down-regulation of hypothalamic pro-opiomelanocortin (POMC) expression after weaning is associated with hyperphagia-induced obesity in JCR rats overexpressing neuropeptide $Y$
}

\author{
Abdoulaye Diané ${ }^{1}$, W. David Pierce ${ }^{2 *}$, James C. Russell ${ }^{1}$, C. Donald Heth ${ }^{3}$, Donna F. Vine ${ }^{1}$, \\ Denis Richard ${ }^{4}$ and Spencer D. Proctor ${ }^{1}$ \\ ${ }^{1}$ Metabolic and Cardiovascular Diseases Laboratory, Alberta Institute for Human Nutrition, Alberta Diabetes Institute, \\ University of Alberta, Edmonton, Canada \\ ${ }^{2}$ Department of Sociology, University of Alberta, H.M Tory Building, Edmonton, Canada, AB T6G $2 H 4$ \\ ${ }^{3}$ Department of Psychology, University of Alberta, Edmonton, Canada \\ ${ }^{4}$ Institut Universitaire de Cardiologie et de Pneumologie de l'Université Laval, Quebec, Canada
}

(Submitted 23 April 2013 - Final revision received 15 August 2013 - Accepted 21 August 2013 - First published online 7 October 2013)

Abstract

We hypothesised that hypothalamic feeding-related neuropeptides are differentially expressed in obese-prone and lean-prone rats and trigger overeating-induced obesity. To test this hypothesis, in the present study, we measured energy balance and hypothalamic neuropeptide Y $(N P Y)$ and pro-opiomelanocortin $(P O M C)$ mRNA expressions in male JCR:LA-cp rats. We compared, in independent cohorts, free-feeding obese-prone (Obese-FF) and lean-prone (Lean-FF) rats at pre-weaning (10 d old), weaning (21-25 d old) and early adulthood (8-12 weeks). A group of Obese-pair-feeding (PF) rats pair-fed to the Lean-FF rats was included in the adult cohort. The body weights of 10-d-old Obese-FF and Lean-FF pups were not significantly different. However, when the pups were shifted from dams' milk to solid food (weaning), the obese-prone rats exhibited more energy intake over the days than the lean-prone rats and higher body and fat pad weights and fasting plasma glucose, leptin, insulin and lipid levels. These differences were consistent with higher energy consumption and lower energy expenditure. In the young adult cohort, the differences between the Obese-FF and Lean-FF rats became more pronounced, yielding significant age effects on most of the parameters of the metabolic syndrome, which were reduced in the ObesePF rats. The obese-prone rats displayed higher NPY expression than the lean-prone rats at pre-weaning and weaning, and the expression levels did not differ by age. In contrast, POMC expression exhibited significant age-by-genotype differences. At pre-weaning, there was no genotype difference in POMC expression, but in the weanling cohort, obese-prone pups exhibited lower POMC expression than the lean-prone rats. This genotype difference became more pronounced at adulthood. Overall, the development of hyperphagia-induced obesity in obese-prone JCR rats is related to POMC expression down-regulation in the presence of established NPY overexpression.

Key words: Obese-prone animals: Weanling JCR rats: Homeostasis: Neuropeptides

Over the last few decades, the incidence of paediatric obesity has increased in both developing and developed countries $^{(1,2)}$. Obesity is characterised by an imbalance between energy intake and energy expenditure, and in children, it is associated with acute and chronic health risks in later life, including $\mathrm{CVD}^{(3)}$ and type 2 diabetes ${ }^{(4)}$. Given the prevalence of obesity, substantial research has been directed at the underlying mechanisms in an effort to discover effective long-term treatment for excessive weight gain and associated chronic disease risk $^{(5)}$. The evidence suggests that genetic differences may help to explain the within-population variation in $\mathrm{BMI}^{(6)}$, together with specific environmental factors $^{(7)}$. In this regard, the use of GM rodent models of obesity aids the investigation of underlying mechanisms.

Animal models of obesity based on mutations in leptin signalling, either at the peptide level ( $o b / o b$ mouse) or at the receptor level $(d b / d b \text { mouse and } \mathrm{fa} / \mathrm{fa} \text { and } c p / c p \text { rats })^{(8)}$, allow investigations into the mechanisms regulating energy balance. Leptin, encoded by the $o b$ gene, is an important mediator of feeding behaviour and energy homeostasis ${ }^{(9)}$. Leptin acts on the hypothalamic regions involved in energy homeostasis, through the leptin receptor. The effects of

Abbreviations: ARC, arcuate nucleus; E EFF, energy efficiency; FF, free-fed; Lean-FF, lean-prone (+/?); NPY, neuropeptide Y; Obese-FF, obese-prone $(c p / c p)$; Obese-PF, pair-fed; POMC, pro-opiomelanocortin; WAT, white adipose tissue.

*Corresponding author: W. D. Pierce, fax +1 7804927196 , email dpierce@ualberta.ca 
leptin on energy balance involve the orexigenic neuropeptide Y (NPY) and agouti-related protein as well as anorexigenic neuropeptides such as pro-opiomelanocortin (POMC) and cocaine-amphetamine-regulated transcript ${ }^{(10)}$.

The JCR:LA-cp rat has the autosomal recessive corpulence $(c p)$ gene, a nonsense mutation in the $O b R$ gene resulting in a premature stop codon in the extracellular domain of the leptin receptor gene ${ }^{(11)}$. Thus, all isoforms of the receptor are affected, leading to a total absence of functional leptin receptors ${ }^{(12)}$. Rats that are heterozygous for the cp trait $(+/$ ?) are lean-prone, while those that are homozygous $(c p / c p)$ display characteristics observed in human obesity - including hyperphagia, hyperinsulinaemia, hyperlipidaemia and atherosclerosis ${ }^{(8,13)}$. These metabolic alterations are well documented in adult rats ${ }^{(8)}$. However, the early onset of these dysfunctions has not been described in the JCR strain.

Epidemiological studies have shown that obesity and associated metabolic complications in adult humans originate, at least in part, early in postnatal life ${ }^{(14)}$. Consistently, studies in rodents have indicated that prenatal and neonatal nutritional environments contribute to adult obesity and related metabolic dysfunctions, which can be further influenced by the post-weaning dietary environment ${ }^{(15,16)}$ and genetic background $^{(17)}$. However, the underlying mechanisms remain unknown.

Nutritional environment during early development has been shown to alter the expression of genes critical to the regulation of energy homeostasis ${ }^{(18)}$. One possibility is that feeding-related neuropeptide genes are altered in obeseprone animals, resulting in the early onset of homeostatic imbalance and predisposition to the development of obesity later in life. Furthermore, studies in rodents indicate that the appetite-regulatory network in the hypothalamus, mostly in the arcuate nucleus (ARC), does not fully develop until the third postnatal week ${ }^{(19)}$, corresponding to the weaning period. Disruption of leptin signalling would be expected to up-regulate NPY expression, which in turn inhibits POMC expression in the $\mathrm{ARC}^{(20)}$, resulting in obesity ${ }^{(21)}$. Beck et $a{ }^{(22)}$ found that, at both post-weaning and adult ages, Zucker (fa/fa) rats display higher NPY and lower POMC expressions in the ARC compared with their lean counterparts, while no difference is observed between the two genotypes during the pre-weaning or suckling period. However, Zucker $(\mathrm{fa} / \mathrm{fa}$ ) rats have a missense mutation in the leptin receptor gene, which diminishes but not completely eliminates the response to leptin ${ }^{(23)}$. As has been noted, JCR rats have a nonsense mutation in the leptin receptor, and it is unclear whether the alterations in NPY and POMC expressions are established before or after weaning in these rats.

In the present study, we hypothesised that in the ARC NPY mRNA levels would be elevated and POMC mRNA levels reduced before weaning in JCR obese-prone pups compared with those in lean-prone littermates. These differences in pre-weanling pups would be exacerbated by weaning and then maintained until adulthood. We also hypothesised that pair-feeding-induced energy restriction ${ }^{(24)}$ would affect feeding-related neuropeptides.

\section{Materials and methods}

\section{Animals}

Separate cohorts of pre-weanling ( $10 \mathrm{~d}$ old; twelve obeseprone $c p / c p$ and twelve lean-prone $+/$ ?), weanling (3 weeks old; eight obese-prone $c p / c p$ and eight lean-prone $+/$ ?) and young adult ( 8 weeks old; sixteen obese $c p / c p$ and eight lean $+/$ ?) male JCR:LA-cp rats were obtained from the breeding colony at the University of Alberta ${ }^{(8)}$. The rats were housed individually in polycarbonate cages $(47 \mathrm{~cm} \times 27 \mathrm{~cm} \times 20 \mathrm{~cm})$ with sterile wood chip bedding and placed under a reversed $12 \mathrm{~h}$ light $-12 \mathrm{~h}$ dark cycle (lights on: 18.00-06.00 hours). Throughout the experiments, the rats had free access to water and were fed as outlined in the experimental procedures section. All food was laboratory chow (LabDiet ${ }^{\text {тм }}$ 5010, PMI Nutrition International, Inc.; metabolisable energy $=14.31 \mathrm{~kJ} / \mathrm{g}(3.42 \mathrm{kcal} / \mathrm{g}))$. Body weight $(\mathrm{g})$ and food intake $(\mathrm{g})$ measurements were taken daily. The care and use of animals were in accordance with the Guidelines of the Canadian Council of Animal Care and subject to prior review and approval by the Animal Care and Use Committee: Health Sciences, University of Alberta.

\section{Experimental procedures}

The experiments were conducted in three independent batches of lean-prone and obese-prone rats of different ages. For the young adult (8-week-old) cohort, obese-prone $(c p / c p)$ rats were matched for body weights and randomly assigned to the free-feeding (Obese-FF, $n$ 8) or the pair-feeding (Obese-PF, $n$ 8) group. Each Obese-PF rat was matched to a lean freefeeding (Lean-FF) littermate and given the same amount of food daily (LabDiet ${ }^{\mathrm{TM}}$ chow 5010; LabDiet). Food intake was measured by subtracting the amount of food uneaten from the fixed amount given to the rat. Although spillage was minimal, when it did occur, the food lost from the cages was collected and added to the total not consumed. Subsequently, food intake was converted to energy (food $(\mathrm{g}) \times 14.31 \mathrm{~kJ} / \mathrm{g}$ $(3.42 \mathrm{kcal} / \mathrm{g}))$, yielding a measure of daily energy consumption. After 4 weeks of feeding, the young adult rats (now 12 weeks old) were transferred to calorimetric chambers for wholebody energy expenditure measurements. Rats belonging to the weanling cohort ( $21 \mathrm{~d}$ old) were assigned to the Obese-FF $(n$ ) and Lean-FF ( $n$ 8) groups on day 22 when obese-prone and lean-prone rats are distinguishable ${ }^{(13)}$ and transferred to calorimetric chambers for energy expenditure assessment before being killed on day 25. Pre-weanling or suckling pups (10 d old) were killed immediately without being subjected to whole-body indirect calorimetry; using sense 5'-ATGAATGCTG-

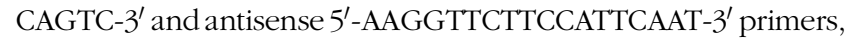
genotyping was carried out using tissue samples collected from the tip of the tail during brain tissue collection, as obese-prone and lean-prone rats are indistinguishable at this age.

\section{Energy expenditure}

Whole-body energy expenditure was measured by indirect calorimetry using a CLAMS system (Columbus Instruments) 
for $24 \mathrm{~h}$ after a $24 \mathrm{~h}$ habituation period, following the manufacturer's instructions. Light and feeding conditions were kept the same as those described above.

\section{Post-mortem analysis}

The rats were killed under anaesthesia with isoflurane after an overnight fast, except the pre-weanling rats, which were killed without fasting. Blood was collected by cardiac puncture. Plasma samples were stored at $-80^{\circ} \mathrm{C}$ until analysis. The rats were immediately perfused intracardially with ice-cold isotonic saline: the brain was removed. Liver mass, lean body mass and white adipose tissue (WAT) mass, the sum of the mass of retroperitoneal, epididymal and subcutaneous depots dissected and weighed, are expressed as a percentage of total body weight.

\section{NPY mRNA expression in hypothalamic arcuate nucleus}

Neuropeptide (NPY and POMC) mRNA in the entire ARC were assessed by in situ hybridisation as described by Doyon et al. ${ }^{(25)}$. The ARC was chosen due to its proximity to the peripheral blood stream and strong responsiveness to peripheral cues to regulate energy balance. The brain tissues were prepared as described previously ${ }^{(26)}$.

\section{Plasma biochemical analysis}

Total plasma cholesterol and TAG assay kits were obtained from Wako USA. Plasma glucose concentration was determined using the glucose oxidase method (Diagnostic Chemical Limited). Insulin and leptin concentrations were assessed using enzyme-linked immunosorbent kits for rodents (insulin kit, Mercodia AB, and leptin kit, LINCO Research). Plasma corticosterone concentration was measured using Milliplex Map (Millipore Corporation).

\section{Statistical analysis}

Results are presented as means with their standard errors and were plotted using SigmaPlot v11 (Systat Software, Inc.). We used two-way ANOVA to test age-by-genotype effects along with follow-up tests using one-way ANOVA for comparison of the three adult groups with the post hoc Bonferroni test for pairwise comparison of means. Student's $t$ test was used to determine statistical differences between the obese and lean groups. All tests and comparisons with $P<0.05$ were considered to be statistically significant.

\section{Results}

\section{Energy intake and body weight}

Fig. 1 shows body weight (Fig. 1(a)), energy intake (Fig. 1(b)) and energy conversion efficiency (Fig. 1(c)) for the freefeeding weanling JCR rats by genotype. The body weights of the Obese-FF and Lean-FF groups were not significantly different on the day of weaning; furthermore, the body weights of (a)

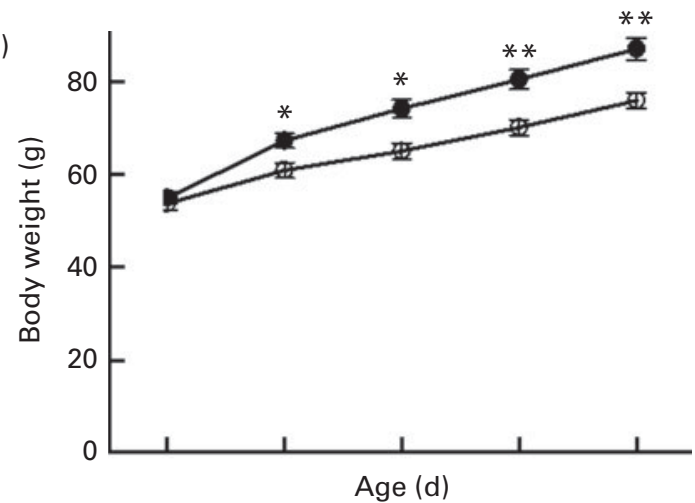

(b)
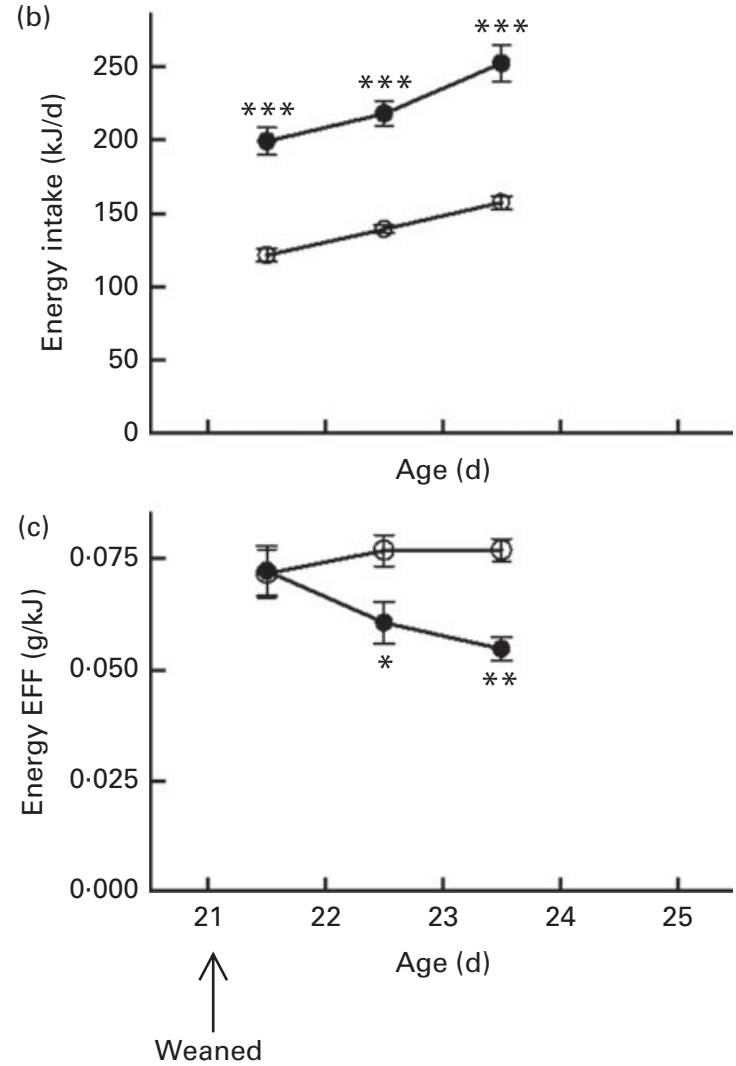

Fig. 1. Effect of genotype on the (a) body weight, (b) energy intake (food consumption expressed as $\mathrm{kJ} / \mathrm{d}$ ) and (c) energy efficiency (EFF, efficiency of conversion to body mass) of weanling JCR:LA-cp rats for three post-weaning days. Values were significantly different from those of the lean-prone $(+/$ ? $)$ rats: ${ }^{*} P<0.05 ;{ }^{\star \star} P<0.01 ;{ }^{* \star \star} P<0.001$. $\multimap$, Lean free-feeding; $-\bullet$, obese free-feeding.

pups in the pre-weanling cohort did not differ significantly by genotype at $10 \mathrm{~d}$ of age (Table 1 ). These findings indicate that lean-prone and obese-prone pups have similar body weights on being shifted to dams' milk during the suckling period.

Weanling rats shifted from dams' milk to chow diet exhibited significantly higher body weights over days $22-25$ in the Obese-FF group than in the Lean-FF group $(P<0.01$; Fig. 1(a)). The energy intake of the Obese-FF group was also higher than that of the Lean-FF group over the postweaning period $(P<0.001$; Fig. 1(b)), but the rate of increase in body weight (regression slopes, Obese-FF group: 6.56 
(SE 0.8) g/d; Lean-FF group: $5.02($ SE 0.72$) \mathrm{g} / \mathrm{d}$ ) was not significantly different. This observation may be related to the lower energy efficiency (E EFF = body weight gain ( $\mathrm{g}$ )/total energy $(\mathrm{kJ})$ ) in the Obese-FF rats (Fig. 1(c)). The E EFF was similar in the two genotypes after weaning (day 21). Thereafter, the Lean-FF rats maintained a constant E EFF, while the ObeseFF rats exhibited a significant decrease in E EFF compared with the Lean-FF rats on days 22 and 23 after weaning $(P<0.05$ and $P<0.01$, respectively).

In the young adult cohort, body weight increased significantly in the three groups during the 4-week feeding period $(P<0 \cdot 001)$. Body weight increased significantly in the Obese$\mathrm{FF}$ rats than in the Lean-FF rats $(P<0 \cdot 001)$ or the Obese-PF rats $(P<0 \cdot 001$; Fig. 2(a)). From 10 to 12 weeks of age, weight gain in the Obese-FF rats $(30.7$ (SE 7.4) g/week) was nearly double that in the Lean-FF rats (16.6 (SE $2 \cdot 8$ ) g/week) and $30 \%$ greater than that in the Obese-PF group (20.3 (SE 4.4) g/week). The Obese-FF rats also maintained significantly higher daily energy intake compared with the Lean-FF or Obese-PF rats $(P<0.01 ;$ Fig. 2(b)). The mean daily energy intake was constant in the Lean-FF rats over the 4-week feeding period, while that in the Obese-FF rats decreased between weeks 10 and $11(P<0 \cdot 01)$ and then remained constant. Over 4 weeks of feeding, total body weight gain in the Obese-PF rats (44.0 (SE $3 \cdot 8)$ g) was nearly $50 \%$ less than that in the Lean-FF group $(87.3$ (SE 3.1) g) $(P<0.001)$. Thus, pair-feeding obese-prone rats to lean-prone rats resulted in a substantial reduction in daily body weight gain.

In the young adult cohort, the E EFF decreased during weeks $8-10$ in the Obese-FF and Lean-FF rats (overall rate of change -33.5 (se 5.9) and $-36.4(\mathrm{se} 5.9) \mathrm{mg} / \mathrm{kJ}$ per $\mathrm{d}$ $(-8.0$ (SE 1.4 ) and -8.7 (SE 1.4$) \mathrm{mg} / \mathrm{kcal}$ per d), respectively, $P<0 \cdot 001)$. The Obese-PF rats exhibited markedly lower E EFF than the Obese-FF or Lean-FF rats $(P=0 \cdot 01)$ on the 1st day of pair-feeding, mirrored by a loss in body weight induced by the initiation of pair-feeding (Fig. 2(c)).

\section{Body composition and plasma biochemical parameters}

In the pre-weanling cohort, despite the same body weight, the percentage of total WAT mass was significantly higher in the obese-prone rats than in the lean-prone rats, with no significant difference being observed in the percentage of lean body mass between the two genotypes (Table 1). In the weanling cohort, the obese-prone rats also had a higher percentage of WAT mass than the lean-prone group $(P<0 \cdot 001)$ and a lower percentage of lean body mass $(P<0 \cdot 001)$.

In the young adult cohort, the percentages of liver mass and total WAT mass were significantly higher in the ObeseFF rats than in their Lean-FF littermates $(P<0 \cdot 01)$; also, the Obese-FF rats had significantly lower lean body mass than the lean-prone rats $(P<0 \cdot 001)$ (Table 1$)$. The Obese-PF rats had significantly lower total WAT mass $(P<0 \cdot 001)$ than the Obese-FF rats, but lean body mass did not differ significantly in both the groups. The two-way ANOVA of age group $x$ genotype revealed a significant main effect of age group on the percentage of total fat mass and lean body mass $(P<0 \cdot 001)$, indicating that the pre-weanling rats had higher 

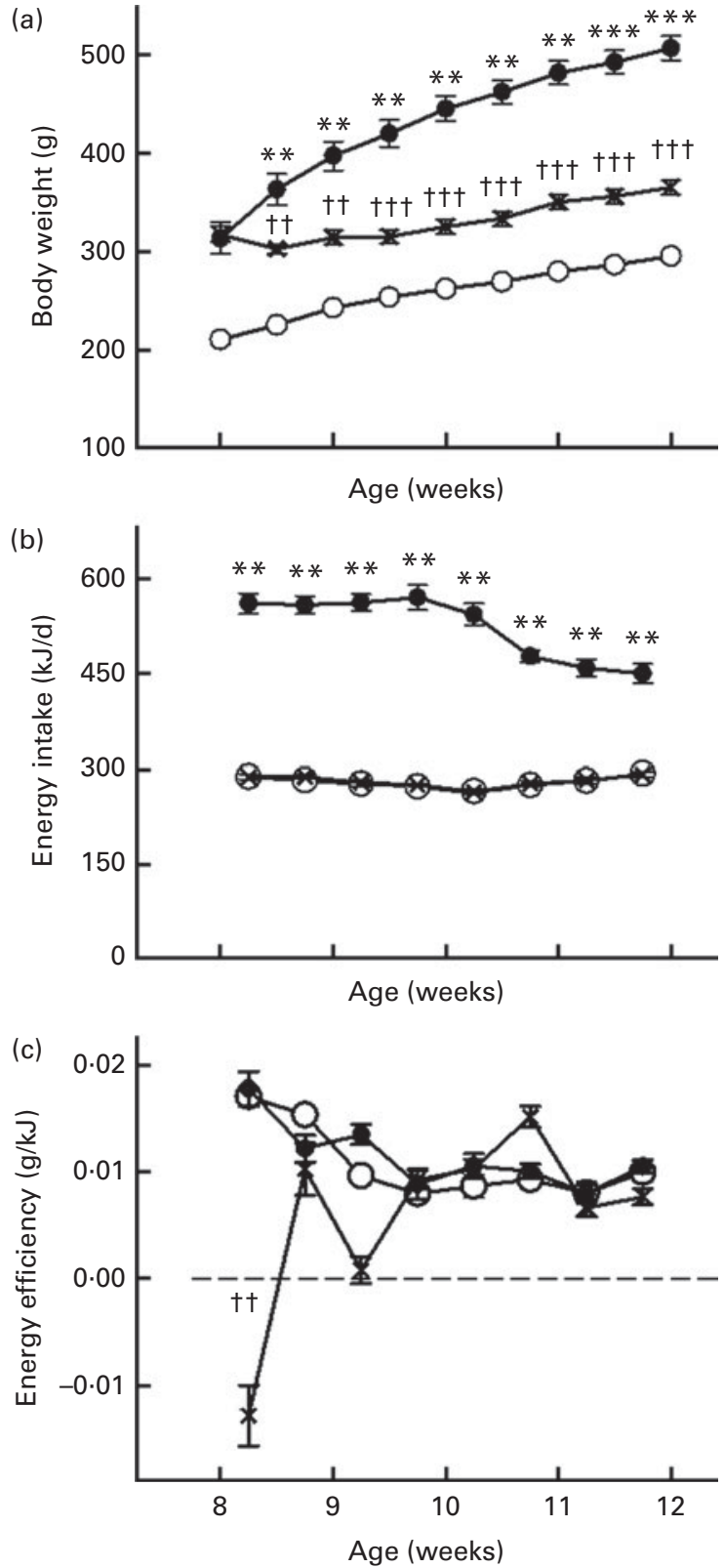

Fig. 2. Effect of feeding groups (lean-prone (+/?)-free-fed (Lean-FF, - ), obese-prone $(c p / c p)$-free-fed (-) and obese-prone $(c p / c p)$-pair-fed (Obese$\left.\mathrm{PF}, x_{-}\right)$) on the (a) body weight, (b) energy intake (food consumption expressed as $\mathrm{kJ} / \mathrm{d}$ ) and (c) energy efficiency (efficiency of conversion to body mass) of JCR:LA-cp rats from 8 to 12 weeks of age (young adults) Statistical differences among the experimental groups are described in the Results section. Note that the variance in energy intake (b) of the Lean-FF and Obese-PF rats was smaller than the size of the symbols and thus not readily visible in the figure. Values were significantly different from those of the Lean-FF rats: ${ }^{\star} P<0.05$; ${ }^{* \star} P<0.01 ;{ }^{* \star \star} P<0.001$. Values were significantly different from those of the Obese-PF rats: $\dagger^{+} P<0.01$; ††† $P<0.001$.

percentages of fat mass and lean body mass than the weanling rats. However, the weanling cohort had lower percentages of fat mass (2.21 (SE 0.17)) and lean mass (34.62 (se 0.53)) than the young adult rats (12.54 (SE 0.37) and 39.87 (SE 0.48)). Thus, the percentage of fat mass decreased between the pre-weaning and weaning groups, but increased in the young adult cohort.
Plasma total cholesterol, TAG and insulin concentrations were significantly lower in the lean-prone rats than in the obese-prone rats, across the age cohorts (Table $1 ; P<0.05$ ). Pair-feeding for a period of 4 weeks significantly decreased plasma total cholesterol and TAG concentrations in young adult Obese-PF rats compared with those in the Obese-FF rats $(P<0.05)$; however, no effect was observed for plasma glucose and insulin concentrations (Table 1).

Interestingly, plasma corticosterone concentrations at the weanling age were significantly higher in the Lean-FF rats than in the Obese-FF rats (Table $1, P<0.05$ ), and this difference was reversed in young adult rats, as the Obese-FF rats had higher concentrations than the Lean-FF rats $(P<0.05)$.

\section{Energy expenditure}

Fig. 3 shows the energy expenditure of rats, either at weaning or young adult ages. Fig. 3(a) shows the RER (relative exchange ratio or RQ), an index of what macronutrients are being preferentially metabolised to produce energy. At both the ages, RER was significantly lower in the Lean-FF rats than in the Obese-FF rats, regardless of the light cycle phase $(P<0.05)$. RER values did not differ significantly between the Obese-FF and Obese-PF young adult rats. Weanling rats of both the genotypes expended more energy than rats in the young adult cohort $(P<0 \cdot 05$; Fig. 3(b)). Fig. 3(b) shows that heat production was higher in the Lean-FF rats than in the Obese-FF rats during both the light and dark phases $(P<0.05)$ at both the ages. The Obese-PF rats exhibited significantly greater heat production than the Obese-FF rats only during the dark phase $(P<0 \cdot 05$; Fig. 3(b)). Locomotor activity (Fig. 3(c)) differed by genotype; in the weanling cohort, the Lean-FF rats were more active than the Obese-FF rats only in the dark phase $(P<0.05)$, and in the young adult cohort, the Lean-FF rats were more active than the Obese-FF rats during both the phases of the diurnal cycle. In the young adult cohort, the Obese-PF group exhibited significantly greater total activity than the Obese-FF rats only in the dark phase $(P<0 \cdot 05$, Fig. 3(b)).

\section{Arcuate nucleus neuropeptide mRNA expression and plasma leptin concentrations}

In Fig. 4(a) and (b), neuropeptide expression levels in the ARC are shown. In the pre-weanling cohort, the lean-prone rats displayed lower $N P Y$ expression than the obese-prone rats $(P<0.05)$. POMC expression was not statistically different between the genotypes. In the weanling cohort, the Lean-FF rats also exhibited lower $N P Y$ mRNA expression than the Obese-FF rats. In the weanling cohort, $P O M C$ expression levels were significantly higher in the lean-prone rats than in the obese-prone rats $(P<0.05)$. In the young adult cohort, $N P Y$ mRNA expression levels tended to be higher in the Obese-FF rats than in the Lean-FF rats, but this difference was not statistically significant $(P=0 \cdot 11)$. In the young adult cohort, $P O M C$ expression in the Obese-FF and Obese-PF rats was significantly lower than that in the Lean-FF rats. Pair-feeding for a period of 4 weeks significantly increased 

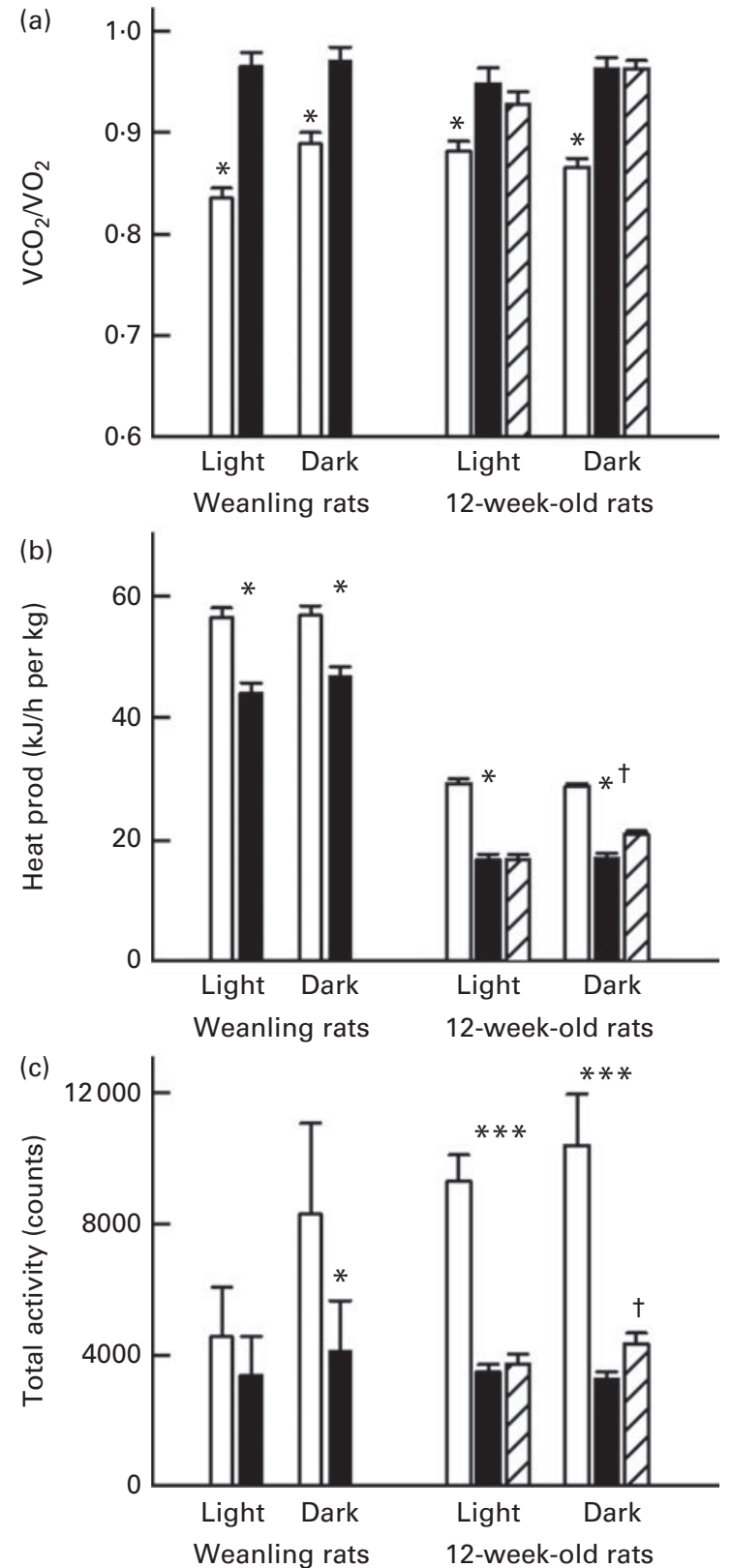

Fig. 3. Effect of feeding groups (lean-prone (+/?)-free-fed (Lean-FF, $\square$ ), obese-prone $(c p / c p)$-free-fed (Obese-FF, $\square$ ) and obese-prone $(c p / c p)$ pair-fed ( $($ ) ) on RER, heat production (prod) and relative activity in the light and dark phases for weanling and 12-week-old JCR:LA-cp rats. Values were significantly different from those of Lean-FF rats: ${ }^{*} P<0.05$; ${ }^{\star *} P<0.01$; ${ }^{\star * \star} P<0.001$. $†$ Values were significantly different from those of the Obese-FF rats $(P<0.05)$.

$N P Y$ expression in the obese-prone rats (Obese-FF $v$. ObesePF; $P<0.001)$. There was no difference in $P O M C$ expression between the Obese-PF and Obese-FF rats. In the lean-prone rats as well as in the obese-prone rats, $N P Y$ expression did not differ significantly by age, but $P O M C$ expression exhibited an age-dependent increase in the lean-prone rats $(P<0.05)$, while it displayed an age-dependent decrease in the obeseprone rats $(P<0 \cdot 02)$.

Plasma leptin concentrations were higher in the obeseprone rats regardless of age. In the pre-weanling cohort, plasma leptin concentrations in the obese-prone pups were five times greater than those in the lean-prone pups $(P<0.01$; Fig. 4(c)). This genotype difference increased substantially with age. Plasma leptin concentrations in the obese-prone rats were twenty-eight times and eighty times higher than those in the lean-prone rats at weaning and adulthood, respectively ( $P<0 \cdot 001$; Fig. 4(c)). Finally, the Obese-PF rats had significantly lower plasma leptin concentrations than the Obese-FF rats $(P<0 \cdot 05)$.

\section{Discussion}

The present study shows, for the first time, that in the ARC POMC expression differs by age and genotype, while NPY expression is only genotype dependent in animals with
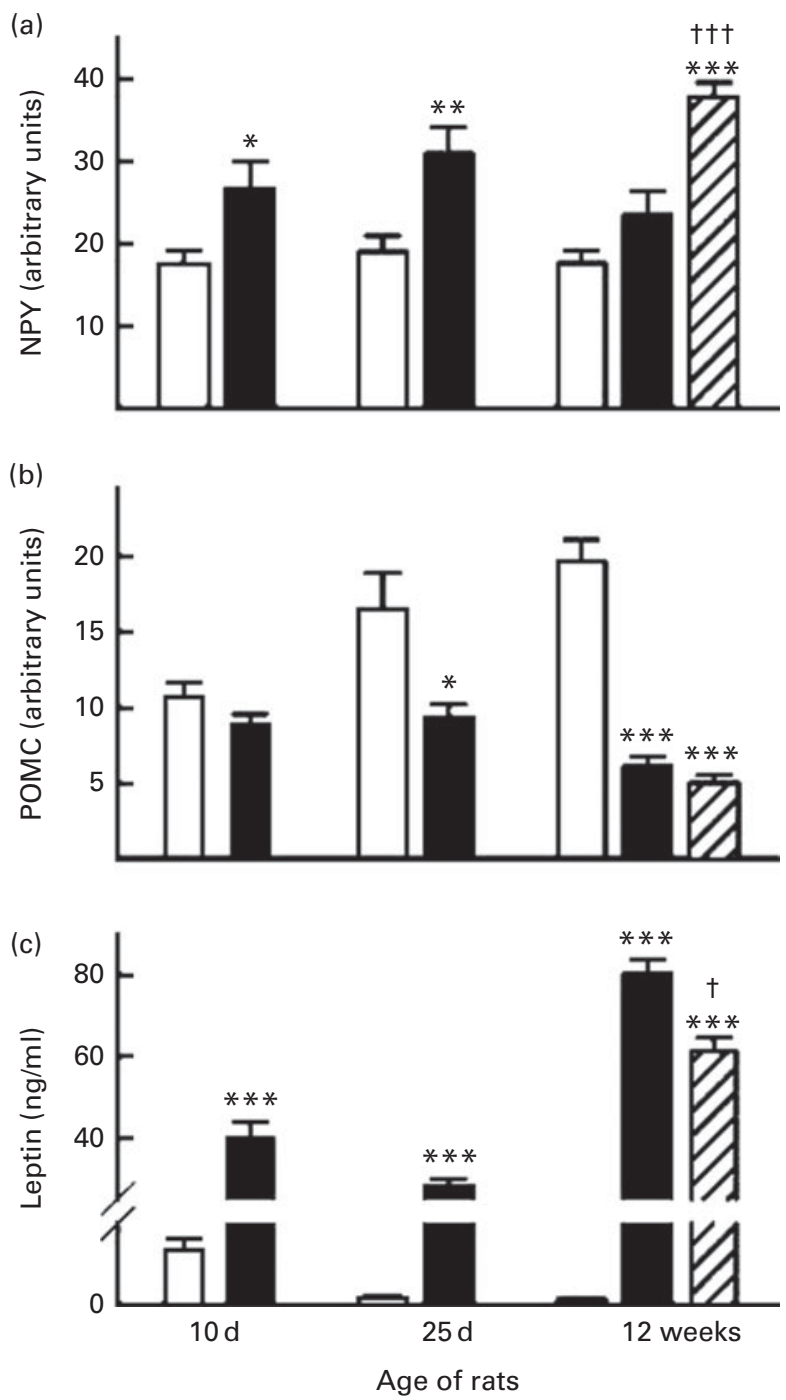

Fig. 4. Effect of feeding groups (lean-prone (+/?)-free-fed (Lean-FF, $\square$ ), obese-prone $(c p / c p)$-free-fed (Obese-FF, $\square)$ and obese-prone $(c p / c p)$ pair-fed $(\nabla)$ ) on the arcuate nucleus neuropeptide expression levels for neuropeptide $Y(N P Y)$ and pro-opiomelanocortin (POMC) and plasma leptin concentrations of pre-weanling, weanling and young adult JCR:LA-cp rats. Values were significantly different from those of the Lean-FF rats: ${ }^{*} P<0.05$; ${ }^{\star \star} P<0.01 ;{ }^{* \star *} P<0.001$. Values were significantly different from those of the Obese-FF rats: $† P<0.05$; $† \dagger P<0.001$. 
a nonsense mutation in the leptin receptor. In contrast, in animals with a missense mutation in the leptin receptor gene (Zucker fa/fa rats), NPY expression is both genotypeand age-dependent ${ }^{(22)}$ - indicating that the onset of NPY expression up-regulation in obese-prone animals is a function of the mutant leptin receptor. At postnatal day 10, obeseprone JCR rats exhibited higher NPY expression than the lean-prone rats, but no difference in POMC expression. At this age, it is difficult to link NPY expression to feeding, as the determination of energy intake (milk consumption) in suckling rats is very difficult. The high fat mass found in the obese-prone rats, however, may be related to the metabolic and anti-thermogenic effects of NPY ${ }^{(27)}$.

The difference in NPY expression by genotype remained in the weanling cohort, but we detected a difference in POMC expression, with obese-prone pups exhibiting downregulation compared with the lean-prone rats. One possibility is that the onset of overeating and body weight gain in obeseprone animals is more related to the down-regulation of the expression of POMC, an anorexigenic peptide, than to the activation of NPY alone. Furthermore, the difference in POMC expression in obese-prone and lean-prone weanling pups coincides with the change from dams' milk to chow diet at weaning. One possibility is that the shift to solid food may combine with genotype to reduce POMC expression at weaning. The results obtained for the young adult cohort support the POMC down-regulation hypothesis of obesity; at this age, the POMC concentrations of the obese-prone rats were further suppressed compared with those of the leanprone rats, coinciding with the onset of obesity and metabolic syndrome in the young adult obese-prone rats. It is possible that the down-regulation of POMC mRNA expression in the ARC is central for the development of obesity in JCR rats, a finding reported previously for tubby mice ${ }^{(28)}$. POMC is also an important anorexigenic regulator of feeding behaviour. In fact, POMC neurons are suppressed by NPY, and NPY-induced feeding behaviour is related to the suppression of POMC signalling ${ }^{(29)}$. One implication is that the down-regulation of POMC expression observed after weaning involves the suppressive effects of NPY on POMC neurons, leading to high energy intake in weanling and young adult obese-prone rats.

Leptin regulates arcuate hypothalamic neurons directly by binding to the ObR receptor long isoform (LRb), activating Janus kinase/signal transducer and activator of transcription 3 (JAK-STAT3) signalling and suppressing the activation of NPY neurons ${ }^{(30)}$. Thus, the early NPY overexpression observed in the pre-weanling obese-prone rats could be linked to the absence of functional leptin receptors. Leptin also induces the expression of uncoupling proteins- $1,-2$ and -3 in the mitochondria, thereby leading to increased thermogenesis ${ }^{(31)}$. In contrast, rodents without leptin receptors, but with intact $N P Y$ and POMC genes, exhibit hyperphagia and decreased thermogenesis ${ }^{(32)}$. However, animals lacking both leptin receptors and $N P Y / P O M C$ genes do not exhibit obesity ${ }^{(33)}$, indicating that functional $N P Y / P O M C$ genes are necessary for the development of obesity. Moreover, a number of studies have reported that diet-induced thermogenesis, accounting for $5-15 \%$ of total daily energy expenditure in humans ${ }^{(32)}$, is impaired in obese-prone subjects ${ }^{(34,35)}$. Thus, overfeeding in weanling and young adult obese-prone rats together with a low thermogenic response may be a contributing factor for energy storage in the $c p / c p$ animals. Glucocorticoid excess, a marker of hypothalamic-pituitary-adrenal axis activity/reactivity ${ }^{(36)}$, has also been observed to be associated with obesity in the present study. As glucocorticoids influence the transcription of hypothalamic neuropeptides ${ }^{(37)}$, the down-regulation of POMC expression in $c p / c p$ animals is probably related to elevated plasma corticosterone concentrations in these animals. The higher plasma corticosterone concentrations of weanling Lean-FF rats compared with the concentrations of the ObeseFF rats is indicative of the high stress induced by maternal separation in lean-prone animals ${ }^{(38)}$. Thus, a functional leptin pathway in the lean-prone rats is associated with the hypothalamic-pituitary-adrenal axis response to maternal separation, but without leptin regulation, obese-prone (ObR absent) rats exhibit a reduced stress response after weaning. As corticosterone concentrations were measured on day 25 , one possibility is that the stress of maternal separation is related to the shift from dams' milk to solid food. When shifted, the obese-prone rats adapted to the change in feeding more rapidly than the lean-prone rats, exhibiting higher energy intake and body weight over the first few days following weaning. This rapid adaptation of the obese-prone rats to the change in feeding would account for the low HPA axis activity in these rats when separated from their mothers.

POMC expression down-regulation is often accompanied by physiological and metabolic changes ${ }^{(20)}$. In the weanling cohort, with free access to food, the high energy consumption of obese-prone rats was associated with body weight gain and increased plasma insulin and lipid concentrations. Furthermore, the percentage of fat mass was greater in the pre-weanling pups than in the weanling pups regardless of the genotype. This age group difference may reflect metabolic and endocrine traits not fully established in neonates ${ }^{(39)}$, making the regulation of energy metabolism inefficient. Under chow diet, from weaning to adulthood, gains in body weight were predominately related to fat mass in the Obese-FF rats, while gains were associated with fat-free mass in the Lean-FF rats. Similar differences have been reported for obese-prone Fischer (F344) rats and Lou obesityresistant rats ${ }^{(40)}$.

A new finding was that the weanling $c p / c p$ pups exhibited lower E EFF than the lean-prone weanling pups. The lower E EFF observed in the weanling Obese-FF pups, despite higher energy intake and body weight, may be related to the energy required to process the excess food. Notably, reduction in E EFF has been observed for rats on a high-fat diet and interpreted as a way to resist the development of obesity ${ }^{(41)}$, occurring mostly during the growth period of $0-30 \mathrm{~d}$.

With regard to pair-feeding, an imposed energy restriction ${ }^{(24)}$, the present results indicate that the young adult $c p / c p$ rats had significantly reduced plasma lipid concentrations. Longterm severe food restriction $(12 \mathrm{~g} / \mathrm{d})$ reduces the plasma TAG concentrations of $c p / c p$ rats (largely in the VLDL fraction) and prevents end-stage $\mathrm{CVD}^{(8)}$. In the present study, we showed that a modest energy restriction also significantly reduces 
plasma cholesterol (15\%) and TAG (62\%) concentrations of Obese-PF rats compared with those of the Obese-FF rats. Low plasma lipid concentrations in the Obese-PF rats were associated with increased NPY and reduced POMC expressions, which are known to trigger food intake ${ }^{(42)}$. However, due to the pair-feeding procedure that makes food availability intermittent, the catabolic pathway is also activated to produce energy, explaining the reduction in WAT mass and TAG concentrations in the Obese-PF rats. The high NPY expression in the Obese-PF rats is also consistent with the increase in the locomotion of these rats, attesting the role of NPY in the regulation of appetitive ingestive behaviour such as locomotion or food-related travel when food is scarce ${ }^{(43)}$. One possibility is that pair-feeding activated the catabolic pathway, explaining the low WAT mass and TAG concentrations in the Obese-PF rats.

Overall, we have shown that in the ARC NPY mRNA expression is up-regulated in pre-weanling obese-prone suckling rats. This $N P Y$ overexpression in obese-prone suckling rats is similar to that in weanling and young adult rats with well-established features of the metabolic syndrome. A difference in POMC expression between lean-prone and obese-prone rats occurs after weaning and it is age dependent. These findings suggest that overeating-induced obesity and associated metabolic syndrome in rats with no functional leptin receptors may be the consequence of the downregulation of POMC expression in the ARC in the presence of established NPY overexpression, suggesting that POMC plays a crucial role in energy homeostasis.

\section{Acknowledgements}

The present study was funded by a grant from the National Sciences and Engineering Research to Council of Canada (NSERC) to W. D. P. and C. D. H.; the Alberta Livestock Industry Development Fund and NSERC supported the research of S. D. P.

The Alberta Livestock Industry Development Fund and NSERC had no role in the design and analysis of the study or in the writing of this article.

The authors' contributions were as follows: A. D., W. D. P. and S. D. P. were responsible for the conception and design of the research; A. D. conducted the experiments; A. D., D. R. and W. D. P. analysed the data and interpreted the results of the experiments; A. D. and J. C. R. prepared the figures; A. D. drafted the manuscript; D. F. V., C. D. H., J. C. R., W. D. P. and S. D. P. edited and revised the manuscript; D. F. V., C. D. H., J. C. R., D. R., W. D. P. and S. D. P. approved the final version of the manuscript.

None of the authors has any conflicts of interest, financial or otherwise, to declare.

\section{References}

1. Lobstein T, Baur L \& Uauy R (2004) Obesity in children and young people: a crisis in public health. Obes Rev 1, 4-104.

2. Popkin BM, Conde W, Hou N, et al. (2006) Is there a lag globally in overweight trends for children compared with adults? Obesity 14, 1846-1853.
3. Must A \& Anderson SE (2003) Effects of obesity on morbidity in children and adolescents. Nutr Clin Care 6, 4-12.

4. Fagot-Campagna A, Pettitt DJ, Engelgau MM, et al. (2000) Type 2 diabetes among North American children and adolescents: an epidemiologic review and a public health perspective. J Pediatr 136, 664-672.

5. Flynn MA, McNeil DA, Maloff B, et al. (2006) Reducing obesity and related chronic disease risk in children and youth: a synthesis of evidence with 'best practice' recommendations. Obes Rev 1, 7-66.

6. Schousboe K, Visscher PM, Erbas B, et al. (2004) Twin study of genetic and environmental influences on adult body size, shape, and composition. Int J Obes Relat Metab Disord 28, 39-48.

7. Qi L \& Cho YA (2008) Gene-environment interaction and obesity. Nutr Rev 66, 684-694.

8. Russell JC \& Proctor SD (2006) Small animal models of cardiovascular disease: tools for the study of the roles of metabolic syndrome, dyslipidemia, and atherosclerosis. Cardiovasc Pathol 15, 318-330.

9. Bates SH \& Myers MG Jr (2003) The role of leptin receptor signaling in feeding and neuroendocrine function. Trends Endocrinol Metab 14, 447-452.

10. Ingvartsen KL \& Boisclair YR (2001) Leptin and the regulation of food intake, energy homeostasis and immunity with special focus on periparturient ruminants. Domest Anim Endocrinol 21, 215-250.

11. Wu-Peng XS, Chua SC Jr, Okada N, et al. (1997) Phenotype of the obese Koletsky (f) rat due to Tyr763Stop mutation in the extracellular domain of the leptin receptor (Lepr): evidence for deficient plasma-to-CSF transport of leptin in both the Zucker and Koletsky obese rat. Diabetes 1997, 513-518.

12. Takaya K, Ogawa Y, Hiraoka J, et al. (1996) Nonsense mutation of leptin receptor in the obese spontaneously hypertensive Koletsky rat. Nat Genet 14, 130-131.

13. Brindley DN \& Russell JC (2002) Animal models of insulin resistance and cardiovascular disease: some therapeutic approaches using JCR:LA-cp rat. Diabetes Obes Metab 4, $1-10$.

14. Breier BH, Vickers MH, Ikenasio BA, et al. (2001) Fetal programming of appetite and obesity. Mol Cell Endocrinol 185, 73-79.

15. Page KC, Malik RE, Ripple JA, et al. (2009) Maternal and postweaning diet interaction alters hypothalamic gene expression and modulates response to a high-fat diet in male offspring. Am J Physiol Regul Integr Comp Physiol 297, R1049-R1057.

16. Kenasio-Thorpe BA, Breier BH, Vickers MH, et al. (2007) Prenatal influences on susceptibility to diet-induced obesity are mediated by altered neuroendocrine gene expression. J Endocrinol 193, 31-37.

17. Levin BE \& Govek E (1998) Gestational obesity accentuates obesity in obesity-prone progeny. Am J Physiol 275 , R1374-R1379.

18. McMillen IC, Adam CL \& Mühlhäusler BS (2005) Early origins of obesity: programming the appetite regulatory system. J Physiol 565, 9-17.

19. Grove KL \& Smith MS (2003) Ontogeny of the hypothalamic neuropeptide Y system. Physiol Behav 79, 47-63.

20. Williams G, Bing C, Cai XJ, et al. (2001) The hypothalamus and the control of energy homeostasis: different circuits, different purposes. Physiol Behav 74, 683-701.

21. Chua SC Jr, Chung WK, Wu-Peng XS, et al. (1996) Phenotypes of mouse diabetes and rat fatty due to mutations in the OB (leptin) receptor. Science 271, 994-996. 
22. Beck B, Burlet A, Bazin R, et al. (1993) Elevated neuropeptide $\mathrm{Y}$ in the arcuate nucleus of young obese Zucker rats may contribute to the development of their overeating. J Nutr 123, 1168-1172.

23. Yamashita T, Murakami T, Iida M, et al. (1997) Leptin receptor of Zucker fatty rat performs reduced signal transduction. Diabetes 46, 1077-1078.

24. Russell JC, Proctor SD, Kelly SE, et al. (2008) Pair feedingmediated changes in metabolism: stress response and pathophysiology in insulin-resistant, atherosclerosis-prone JCR:LA-cp rats. Am J Physiol Endocrinol Metab 294, E1078-E1087.

25. Doyon C, Denis RG, Baraboi ED, et al. (2006) Effects of rimonabant (SR141716) on fasting-induced hypothalamicpituitary-adrenal axis and neuronal activation in lean and obese Zucker rats. Diabetes 55, 3403-3410.

26. Poulin AM \& Timofeeva E (2008) The dynamics of neuronal activation during food anticipation and feeding in the brain of food-entrained rats. Brain Res 1227, 128-141.

27. Godbole V, York DA \& Bloxham DP (1978) Developmental changes in the fatty (fafa) rat: evidence for defective thermogenesis preceding the hyperlipogenesis and hyperinsulinaemia. Diabetologia 15, 41-44.

28. Guan XM, Yu H \& Van der Ploeg LH (1998) Evidence of altered hypothalamic pro-opiomelanocortin/neuropeptide Y mRNA expression in tubby mice. Brain Res Mol Brain Res 59, 273-279.

29. Kageyama H, Takenoya F, Hirako S, et al. (2012) Neuronal circuits involving neuropeptide $\mathrm{Y}$ in hypothalamic arcuate nucleus-mediated feeding regulation. Neuropeptides $\mathbf{4 1 7 9}$, $103-105$

30. Dhillon SS, McFadden SA, Chalmers JA, et al. (2011) Cellular leptin resistance impairs the leptin-mediated suppression of neuropeptide $\mathrm{Y}$ secretion in hypothalamic neurons. Endocrinology 152, 4138-4147.

31. Scarpace PJ, Matheny M, Pollock BH, et al. (1997) Leptin increases uncoupling protein expression and energy expenditure. Am J Physiol 273, E226-E230.

32. Luo N, Marcelin G, Liu SM, et al. (2011) Neuropeptide Y and agouti-related peptide mediate complementary functions of hyperphagia and reduced energy expenditure in leptin receptor deficiency. Endocrinology 152, 883-889.

33. Schutz Y, Bessard T \& Jéquier E (1984) Diet-induced thermogenesis measured over a whole day in obese and nonobese women. Am J Clin Nutr 40, 542-552.

34. Segal KR, Edaño A \& Tomas MB (1990) Thermic effect of a meal over 3 and $6 \mathrm{~h}$ in lean and obese men. Metabolism 39, 985-992.

35. Segal KR, Edaño A, Blando L, et al. (1990) Comparison of thermic effects of constant and relative caloric loads in lean and obese men. Am J Clin Nutr 51, 14-21.

36. Dallman MF, Akana SF, Pecoraro NC, et al. (2007) Glucocorticoids, the etiology of obesity and the metabolic syndrome. Curr Alzheimer Res 4, 199-204

37. Liu L, Song Z, Sheikhahmadi A, et al. (2012) Effect of corticosterone on gene expression of feed intake regulatory peptides in laying hens. Comp Biochem Physiol B Biochem Mol Biol 162, 81-87.

38. Yoo SB, Ryu V, Park EY, et al. (2011) The arcuate NPY, POMC, and CART expressions responding to food deprivation are exaggerated in young female rats that experienced neonatal maternal separation. Neuropeptides 45, 343-349.

39. Hammon HM, Steinhoff-Wagner J, Schönhusen U, et al. (2012) Energy metabolism in the newborn farm animal with emphasis on the calf: endocrine changes and responses to milk-born and systemic hormones. Domest Anim Endocrinol 43, 171-185.

40. Blum JW \& Hammon H (1999) Endocrine and metabolic aspects in milk-fed calves. Domest Anim Endocrinol 17, 219-230.

41. Abdoulaye D, Wetzler S, Goubern M, et al. (2006) Comparison of energy balance in two inbred strains of rats: Fischer F344 prone to obesity and Lou rats resistant to obesity. Physiol Behav 87, 245-250.

42. Henry M, Ghibaudi L, Gao J, et al. (2005) Energy metabolic profile of mice after chronic activation of central NPY Y1, Y2, or Y5 receptors. Obes Res 13, 36-47.

43. Nergårdh R, Ammar A, Brodin U, et al. (2007) Neuropeptide Y facilitates activity-based-anorexia. Psychoneuroendocrinology 32, 493-495 